\title{
Violations of local equilibrium and linear response in classical lattice theories
}

\author{
Kenichiro Aoki* and Dimitri Kusnezov ${ }^{\dagger}$ \\ * Dept. of Physics, Keio University, \\ 4-1-1 Hiyoshi, Kouhoku-ku, Yokohama 223-8521, Japan \\ ${ }^{\dagger}$ Center for Theoretical Physics, Sloane Physics Lab, Yale University, \\ New Haven, CT 06520-8120, U.S.A.
}

\begin{abstract}
We study the dynamics of $\phi^{4}$ theory and the FPU $\beta$ model under thermal gradients, from first principles. We analyze quantitatively how local equilibrium and linear response are violated, paying special care to how we find observables that unambiguously display these violations. Relations between these quantities to equations of state are also examined. Further, we discuss how we can approach similar dynamical problems in continuum quantum field theory. We analyze how close we are to obtaining the continuum results.
\end{abstract}

\section{INTRODUCTION}

Non-equilibrium dynamics appears in almost all areas of physics, from physics of the early universe, heavy ion collisions to transport in matter. In many of these situations, we would like to find out how physical quantities behave in various quantum field theories under non-equilibrium conditions. In an ideal world, we would like to analyze their dynamics from first principles, with no approximations. Let us first discuss what this entails: We would use no dynamical assumptions, the computations will be fully non-perturbative, transport properties will not be restricted only to linear response and the non-equilibrium properties of the system arise dynamically if some non-equilibrium conditions are imposed at the boundaries. These are some of the requirements that need to be satisfied.

In practice, this is too ambitious. Usually, we resort to computations of the Green-Kubo formulas for the transport coefficients or use the Boltzmann equation, which is truncated from the full BBGKY hierarchy. The equations are solved essentially in equilibrium so that the region of validity of the results are unclear, not to mention that the results can not be applied to situations far from equilibrium. Even then, further approximations need to be made. For instance, the transport coefficients of $\phi^{4}$ theory to leading order in the coupling was completed only recently [1,2]. The reason for this is clear; the computations are quite involved and results can not be obtained in closed form, except for its asymptotic behaviors.

In this work, we study the non-equilibrium dynamics of two types of theories, $\phi^{4}$ theory and the FPU $\beta$ model, in $D=1,2,3$ spatial dimensions. We shall work with classical theories on the lattice and from first principles. In particular, we shall make no dynamical assumptions and our computations will be fully non-perturbative. While the $\phi^{4}$ theory has a bulk limit, the FPU $\beta$ model does not, and the temperature dependence of the transport coefficients qualitatively differs. It hardly needs to be mentioned, but the $\phi^{4}$ theory is a classic prototypical field theory and the FPU $\beta$ model is another classic model which has been studied widely. These distinct theories in various dimensions should allow us to gauge how applicable the results are to more general theories. Putting these systems under weak and strong thermal gradients, we shall study how we can measure deviations from local equilibrium and analyze its behavior quantitatively. We also examine the validity of the linear response law and analyze its violations quantitatively. Further, we study the relations of these violations to the thermodynamic properties of the systems, such as the equations of state. We then discuss how to extend these results to quantum field theories.

The main reason we can analyze the systems from first principles is because we are working with classical systems on a finite lattice (of varying sizes). In the classical theory, the problem essentially reduces to computing the solutions to the equations of motion for the degrees of freedom of the system. We can perform this task without any approximations such as perturbation theory by numerically integrating the equations of motion. While the behavior of a classical system is different from that of a quantum system, the latter often reduces to the former under certain conditions, such as for high temperatures [3]. Also, the classical theory can play a major role in obtaining the results in the full quantum theory, as can be seen from the results in the electroweak theory at finite temperatures 沺. Furthermore, we believe that one should understand the behavior of classical systems first, before we can hope to understand the full quantum theory. We work with lattice theories which we can think of as a real lattice as in solids. We can also

${ }^{*}$ E-mail: ken@phys-h.keio.ac.jp

${ }^{\dagger} \mathrm{E}$-mail: dimitri@nst.physics.yale.edu 
think of the lattice as a discrete approximation of the continuum theory, which is quite natural and corresponds to a theory with a cutoff. Of course, it hardly needs to be mentioned that the behavior of classical lattice systems is of interest on its own right!

\section{THE SYSTEMS}

The Hamiltonians of the models we study are

$$
H=\sum_{\mathbf{r}}\left[\frac{1}{2} \pi_{\mathbf{r}}^{2}+\frac{1}{2}(\nabla \phi)_{\mathbf{r}}^{2}+V\left(\phi_{\mathbf{r}}\right)\right], \quad V_{\phi^{4}}\left(\phi_{\mathbf{r}}\right)=\frac{\phi_{\mathbf{r}}^{4}}{4}, \quad V_{F P U}\left(\phi_{\mathbf{r}}\right)=\frac{(\nabla \phi)_{\mathbf{r}}^{4}}{4}
$$

in $1-3$ spatial dimensions. The sum is taken over all lattice points $\mathbf{r}$ and $\nabla \phi_{\mathbf{r}}$ is the lattice gradient. As should be clear, the $\phi^{4}$ theory can be thought of as a discretized version of the standard continuum $\phi^{4}$ theory. The mass has been set to zero for simplicity here, although we have also studied cases where the mass is non-zero.

In this work, we are interested in the steady state properties of the systems under thermal gradients. As such, we apply thermostats at the boundaries $x=0, L$ and impose periodic boundary conditions for the other directions for $D=2,3$. It is important to note that the thermostats are applied only at the ends so that in the interior, $0<x<L$, the degrees of freedom are solely those of the $\phi^{4}$ theory or the FPU model. The thermostats we use are generalized variants of the thermostats of Nosé and Hoover [5,6]. Explicit forms of equations of motion including the thermostat degrees of freedom can be found in [7,8]. The thermostats are deterministic, rather than stochastic, and the thermostats effectively impose thermal distributions at the thermostatted sites when time averaged. A physical observable, $\mathcal{O}$, such as correlation functions or currents, is computed by sampling them along the classical trajectory of the whole system in the phase space and taking its time average, $\langle\mathcal{O}\rangle$. We studied lattice sizes in the $x$ direction up to $L=10000$ for $D=1$ and up to $L=2000$ and transverse lattice sizes of $L_{\perp}=3 \sim 20$ when $D=2,3$.

Since we will be interested in thermal transport, we shall need an expression for the energy flow,

$$
\mathcal{T}_{\phi^{4}}^{0 x}=-\pi_{\mathbf{r}} \nabla_{x} \phi_{\mathbf{r}}, \quad \mathcal{T}_{F P U}^{0 x}=-\left(\pi_{\mathbf{r}} \nabla_{x} \phi_{\mathbf{r}}\right)\left[1+(\nabla \phi)_{\mathbf{r}}^{2}\right]
$$

which satisfies the usual continuity equation.

\section{NEAR EQUILIBRIUM BEHAVIOR}

When the two end point temperatures, are equal, $T_{1}=T_{2}$, the interior of the system thermalizes at the same temperature. This can be explicitly confirmed by checking that the momentum distribution is Maxwellian. The local temperature is defined by $T(\mathbf{r})=\left\langle\pi_{\mathbf{r}}^{2}\right\rangle$, which is called the ideal gas temperature. This is well defined and is a robust local measure, since the Hamiltonians in (11) are quadratic in $\pi_{\mathbf{r}}$ and $\pi_{\mathbf{r}}$ interact with the neighboring sites only indirectly through $\phi_{\mathbf{r}}$.
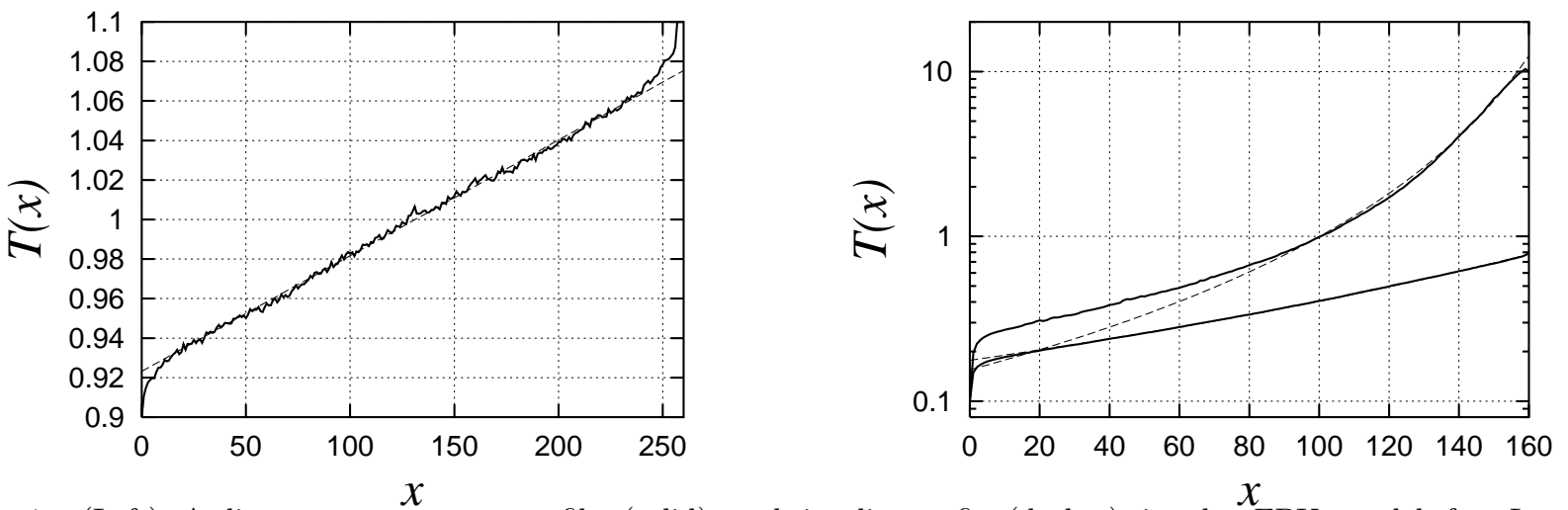

FIG. 1. (Left) A linear temperature profile (solid) and its linear fit (dashes) in the FPU model for $L=260$, $\left(T_{1}, T_{2}\right)=(0.9,1.1), D=1$. (Right) Curved profiles (solid) in $\phi^{4}$ theory with the linear response predictions (dashes) for $L=160,\left(T_{1}, T_{2}\right)=(0.1,0.8),(0.1,10), D=1$. We see that the linear response prediction is applicable for the smaller gradient case, but not for the other. 
When we make the two boundary temperatures slightly different, we recover a straight temperature profile which can be well described by the Fourier's law,

$$
\left\langle\mathcal{T}^{0 x}\right\rangle=-\kappa(T) \nabla_{x} T
$$

as can be seen in Fig. 11(left).1 By choosing various small temperature differences around the same central temperature, we directly extract the thermal conductivity $\kappa$ for a given temperature in each system at each lattice size from measurements of the energy flow and the temperature gradient, using (3). In the $\phi^{4}$ theory, we find a bulk limit exists for $\kappa$, namely the limit $\kappa(L \rightarrow \infty)$ exists [7,8]. In the FPU model, the bulk limit does not exist [10,11. The results can be summarized for the $\phi^{4}$ theory as (see Fig. 2 (left))

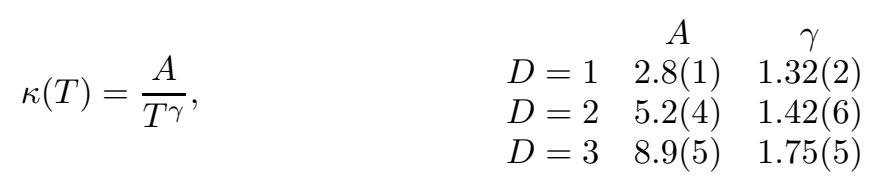

For the FPU model, the behavior is more complicated as can be seen in Fig. 2 (right); $\kappa$ depends on $\mathrm{L}$ and $T$ dependence is no longer a simple power. The $L$ dependence is compatible with a simple power law and the asymptotic behavior

$$
\kappa \simeq\left\{\begin{array}{ll}
1.2 L^{\delta} T^{-1} & (T \lesssim 0.1) \\
2 L^{\delta} T^{1 / 4} & (T \gtrsim 50)
\end{array}, \quad \delta=0.37(3)\right.
$$

can be understood from scaling arguments [1].
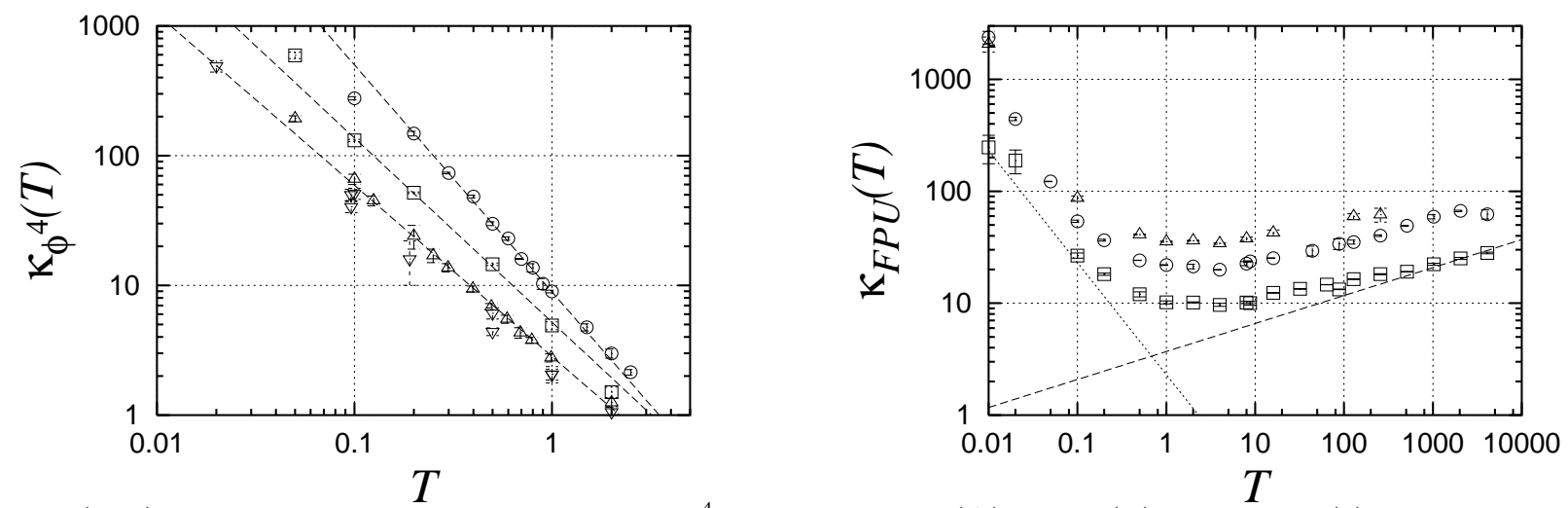

FIG. 2. (Left) Temperature dependence of $\kappa$ for the $\phi^{4}$ theory in $D=1(\triangle) D=2(\square)$, and $D=3(\circ)$ with the power laws of (4) in dashes. The Green-Kubo results for $D=1$ are also shown $(\nabla)$. (Right) Behavior of $\kappa$ for the $D=1$ FPU model for $L=16(\square), L=128(\circ)$ and $L=512(\triangle)$. The asymptotic behaviors of $1 / T$ (dots) and $T^{1 / 4}$ (dashes) are also plotted. The $\phi^{4}$ theory has a bulk limit, whereas the FPU model does not.

We can also compute the thermal conductivity independently of the Fourier's law (3) through the integral of the autocorrelation of the energy current using the Green-Kubo formula. The results agree with the direct computation from Fourier's law. For $D=1 \phi^{4}$ theory, the Green-Kubo results are shown in Fig. 2 (left) along with those from direct computations [9]. We note in passing that the applicability of the Green-Kubo formalism is regarded as being most subtle for lower dimensional systems due to the existence of persistent correlations [12].

As we make the temperature gradients larger, the temperature profiles become visibly non-linear, as in Fig. 11 (right). This does not necessarily signal the breakdown of linear response. (ASince $\kappa$ depends on the temperature ( $c f$. Fig. 2), as the temperature changes, so does the gradient, and it can do so within the same system [13]. This reasoning leads to the following formula for the temperature profile by applying the linear response law (3) locally [7].

\footnotetext{
${ }^{1}$ While it will not be discussed here, in general, temperature jumps will arise at the boundaries which can be understood 9 . ${ }^{2}$ By "linear response", we refer strictly to the validity of (3). In particular, we do not presume that the existence of a bulk limit.
} 


$$
T(x)=T_{1}\left[1-\frac{x}{L}+\left(\frac{T_{2}}{T_{1}}\right)^{1-\gamma} \frac{x}{L}\right]^{\frac{1}{1-\gamma}}
$$

Here, we assumed that the temperature dependence of the thermal conductivity can be described by $\kappa(T)=A T^{-\gamma}$, which is true for the $\phi^{4}$ theory and for most temperature regions in the FPU model. It should be noted that the profile has the scaling behavior that it depends on $x$ only through $x / L$. This formula for the profile works quite well when the we are not too far from equilibrium. For example in Fig. 1 (right), the theoretical curve (6) along with the profile is presented and we see that it provides an excellent description of the curved temperature profile for not so large gradients. We shall turn to the question of quantifying how far we are from equilibrium and how good the description is, in the next section.

\section{BREAKDOWN OF LOCAL EQUILIBRIUM AND LINEAR RESPONSE}

When the system moves further away from equilibrium, the formula (6) is no longer adequate to describe the temperature profile, as in Fig. 1 (right). One reaction might be to employ some kind of 'non-linear' response theory. However, we would first like to understand the overall behavior of the system quantitatively and judge whether such a description is valid. To this end, we would like to also assess if local equilibrium is satisfied so that for instance, the temperature is well defined. For this, we need an explicit measure of how well the local equilibrium holds, which we find in the cumulants of $\pi_{\mathbf{r}}$. Since the Hamiltonian of the system (11) is quadratic in $\pi_{\mathbf{r}}$, the thermal distribution should be Maxwellian if local equilibrium holds. The cumulants provide an unambiguous local measure of local equilibrium breakdown. We shall also need a local measure of how "far" we are from equilibrium and for this, we use $\nabla_{x} T / T$. We note that it is natural to use the rescaled gradient here. This is quite evident in Fig. 目 (right); the linear response prediction is less applicable in the small $T$ region, wherein $\nabla_{x} T$ is smaller but $\nabla_{x} T / T$ is larger.

Before proceeding further, we would like to discuss the general picture: We find that a physical observable, $\mathcal{A}$, generically deviates from its local equilibrium value in a manner

$$
\delta_{\mathcal{A}} \equiv \frac{\delta \mathcal{A}}{\mathcal{A}}=C_{\mathcal{A}}\left(\frac{\nabla_{x} T}{T}\right)^{2}+C_{\mathcal{A}}^{\prime}\left(\frac{\nabla_{x} T}{T}\right)^{4}+\ldots
$$

While this seems quite natural since the intrinsic behavior should not depend on the direction of the gradient, it has not been established analytically. Furthermore, the behavior of the coefficients is more subtle than what one would expect, as we shall see below.

Let us analyze first the violations of local equilibrium and linear response. For deviations from local equilibrium, we use the observable, $\left\langle\left\langle\pi^{4}\right\rangle\right\rangle / T^{2}$ where the fourth cumulant is $\left\langle\left\langle\pi^{4}\right\rangle\right\rangle \equiv\left\langle\pi^{4}\right\rangle-3\left\langle\pi^{2}\right\rangle^{2}$. Likewise, for linear response, we use the observable $\left(\mathcal{T}^{0 x}-\kappa \nabla_{x} T\right) / \kappa \nabla_{x} T$. We show some of the results in Fig. 3. These computations need to be performed with care since we are looking at differences.

From these analyses, we extract the coefficients $C_{L E}, C_{L R}$ in (7) for local equilibrium and linear response, respectively. $C_{L E}, C_{L R}$ can in general depend on $T$ and $L$. We have computed these coefficients at various $T$ and $L$ for both the $\phi^{4}$ theory and the FPU model in various dimensions. While we do not have yet a global picture of how these coefficients behave, a few comments are in order. For the $\phi^{4}$ theory, which has a bulk limit, we would naively expect that these coefficients will be independent of $L$. However, this turns out to be not the case. For instance, at $T=1, C_{L E}=a L$ with $a=3,2,4$ for $D=1,2,3$. There is thus a strong dependence on the size of the system, so that a simple local understanding of (7) does not seem possible. In general, both for the $\phi^{4}$ theory and for the FPU model in $D=1,2,3$ and for various $L$, we find that $\delta_{L E} \sim \delta_{L R}$. This means, in particular, that a simple non-linear response theory is not applicable to these systems since the breakdown of local equilibrium also needs to be considered simultaneously. We note that a priori, it could have been such that $\delta_{L E}$ was much smaller that $\delta_{L R}$, in which case a non-linear response theory would have seemed quite appropriate. Generically, we find the dependence of $C_{L E}, C_{L R}$ on $L$ to be close to linear, and the temperature dependence to be weak, which supports the rescaled gradient $\nabla_{x} T / T$ as a natural measure of how far we are from equilibrium. We have further computed the deviations in the equations of state for pressure and local energy density from local equilibrium relations. We find that the deviations can again be understood using the description in (7). 


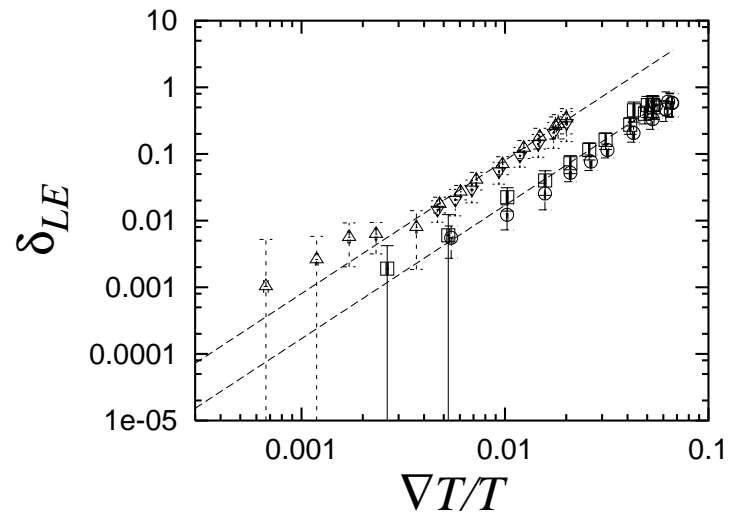

FIG. 3. Violations of local equilibrium and linear response. $L=16, T=8.8(\square), T=88(\circ), L=64, T=8.8(\triangle), T=88(\nabla)$ together with the quadratic behavior (dashes). $L$ dependence is obvious but $T$ dependence is weak. (Right) $\delta_{L R}$ in $\phi^{4}$ theory, $D=1$ at $T=1$ for $L=40$ (०) and $L=80$ ( $\square$ ) along with the quadratic behavior (dashes). $L$ dependent behavior is evident.

\section{TOWARDS QUANTUM FIELD THEORY IN NON-EQUILIBRIUM}

As we have seen in the previous sections, in classical lattice systems, we can ask practically any question regarding the physics behavior of non-equilibrium systems and obtain an answer, at least under steady state conditions. We are not restricted to weak coupling, nor do we have to assume linear response theory and we can be arbitrarily far from equilibrium. Clearly, we want to extend this situation to quantum field theories. Ideally, we would like to solve quantum dynamics in a similar fashion, leading to the solution of quantum field theory in non-equilibrium, in the continuum limit. This is, of course, too ambitious at this point; even for moderately large systems (say $\sim 100$ degrees of freedom), it is computationally prohibitive to carry out such a program. Our goal here will be more modest; we would first like to apply the results of the classical theory to quantum field theory and find out how they fit in. We shall see that even this task is quite non-trivial. Typically, classical results are applicable to quantum theories in some region or in some limit [3.4]. At first sight, the prospect seems quite promising for the lattice $\phi^{4}$ theory; amongst other features, the theory has a bulk limit with the behavior appearing already for system sizes of order hundred or less for moderate temperatures and the temperature profile (6) has a smooth continuum limit.

However, in practice, we immediately encounter an obvious problem: when one tries to take the naive continuum limit by taking the lattice spacing to zero, quantities such as the energy density, thermal conductivity diverge. They diverge for the trivial reason that the system will have an infinite number of oscillators in the unit volume. This is the "Rayleigh-Jeans" problem, known from the early days of the 20th century which afflicts all classical theories in the continuum. While it might seem that this will make it impossible to obtain finite results in any classical continuum theory at finite temperatures, it has been demonstrated that one can obtain finite results for physical observables in the classical continuum theory that matches with the quantum results in the appropriate region [4. 14]. This is done by renormalizing the theory classically using the appropriate matching conditions.

Next point to be resolved before we can start applying our results to quantum field theory is the continuum limit. How far are we from the continuum limit and how do we know? While we do not have a definitive answer to this difficult question, we can study the physical mass in lattice units, $m_{p h} a$, which effectively measures how big our lattice mesh is in physical units. (The lattice size, $a$, has been set to one here.) To avoid lattice artifacts, we would like this quantity to be small. The physical mass can be measured from the correlation function as

$$
\left\langle\phi(0) \sum_{\mathbf{r}_{\perp}} \phi\left(x, \mathbf{r}_{\perp}\right)\right\rangle \sim e^{-m_{p h}|x|}
$$

where $\mathbf{r}_{\perp}$ denotes the coordinates transverse to $x$. In Fig. A (left), we study the correlation function averaged in the transverse direction. We see that the correlation function indeed does decay exponentially, which allows us to extract the physical mass. We find, as in Fig. 国(right), that the physical mass has a temperature dependence which we find to be

$$
m_{p h}= \begin{cases}0.97(2) T^{0.30(1)} & D=1 \\ 0.69(5) T^{0.44(3)} & D=3\end{cases}
$$


To put things in perspective, in lattice QCD, the state of the art simulations are typically run with $m_{p h} a=0.1 \sim 0.5$. So, for $T \lesssim 1$, we should be able to overcome lattice artifacts.
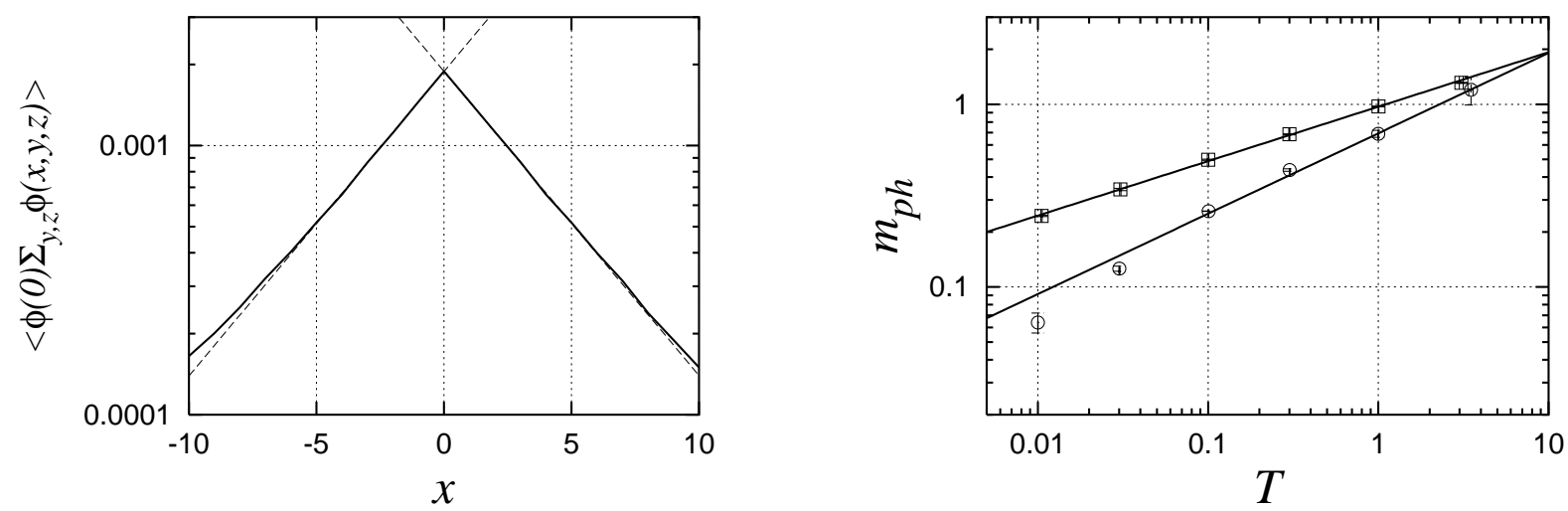

FIG. 4. (Left) The behavior of the correlation function in (8) (solid) and its exponential behavior (dashes) for the $\phi^{4}$ theory. The lattice size is $23 \times 10 \times 10$ at $T=0.1$ in this example. (Right) The behavior of $m_{p h}$ with respect to $T$ for $D=1$ ( $\square$ ), $D=3$ (o). The power behavior (solid) describes the results quite well.

One more aspect needs to be addressed: In the continuum limit, when the lattice no longer exists and the theory becomes relativistic, we have to decide relative to what reference frame we have transport in the bulk. Here, a natural situation is provided if we consider a system with more conserved charges. For instance, it is quite straightforward to extend our analysis to a complex scalar field theory with $\mathrm{U}(1)$ symmetry. In this case, we can address energy transport in the system with no net charge flow, which would allow us to extract the thermal conductivity in the usual sense. Since our methods can be used in practically any classical lattice system, we should be able to address the problem of relativistic transport, with judicious choice of models.

While more work is definitely needed to apply our results to the appropriate regimes of quantum field theory, none of the obstacles seems insurmountable. The lattice artifacts should be reasonably small for $T \lesssim 1$, the divergences of the classical continuum theory can be overcome and the relativistic limit is quite compatible.

\section{DISCUSSIONS}

In summary, we have been able to obtain the physical behavior of $\phi^{4}$ theory and the FPU $\beta$ model under thermal gradients, near and far from equilibrium. Close to equilibrium, we see that linear response theory works well and local equilibrium description is applicable. As we move away from equilibrium, linear response breaks down, but so does local equilibrium. We have measured quantitatively how much they deviate from equilibrium values and they are rather similar in all the systems we studied. The deviation of these quantities from the equilibrium behavior is quadratic in the thermal gradient, as in (7). This behavior is rather generic in the systems we studied, but no analytic explanation of this behavior is known. It is interesting to note that in the non-linear dependence of the viscosity on the shear rate, it was initially argued analytically and further found numerically that the behavior is not quadratic but rather has a non-analytic behavior with a power of $3 / 2$ [15]. Research in this area is still in progress and the question whether the dependence is analytic still seems quite open [16].

We have further discussed how to apply our results to quantum field theory. Obviously, our results do not incorporate essentially quantum behavior so that they can be applied only to certain regimes in the quantum theory. Even though the task is non-trivial, we found that the program is quite feasible.

Much more needs to be done: While we can answer practically any question thrown at us regarding the steady state behavior of classical lattice systems under thermal gradients, the results by themselves lack analytic understanding. We have tried to fill in the missing links by analyzing these results, but we feel that a deeper understanding of the phenomena and their relation to the intrinsic non-equilibrium dynamics is highly desirable. For instance, as we mentioned above, even the quadratic behavior of the deviations from equilibrium is something that has not been established analytically in any model. While this behavior might be intuitive and understandable, the fact that we have the breakdown of local behavior in these deviations is quite subtle and needs to be explained. In applying the classical results to the quantum theory, we still need to complete the program. Furthermore, if one wanted to perform even moderately large scale quantum simulations from first principles, how to approach it is still unclear. Also, even 
in classical systems, we have not dealt with transient phenomena, which is an interesting and important avenue of study. We analyzed the steady state physics as a first step, but many non-equilibrium phenomena, such as the theory of the early universe or heavy ion collisions, are intrinsically time dependent. We feel that there is still much more interesting physics to be uncovered in the area of non-equilibrium systems, even classically.

Acknowledgements: We would like to thank Gert Aarts, Jan Smit and Larry Yaffe for stimulating discussions regarding the material in the last section.

[1] Yu. S. Gangnus, A.V. Prozorkevich, S.A. Smolyanskii JETP Lett. 28 (1978) 347; A. Hosoya, M. Sakagami, M. Takao, Ann. Phys. (NY) 154 (1984) 229.

[2] S. Jeon, Phys. Rev. D52 (1995) 3591; S. Jeon, L. Yaffe, Phys. Rev. D53 (1996) 5799.

[3] See, for instance, M. Le Bellac, Thermal Field Theory, Cambridge University Press (1996)

[4] D.Yu. Grigoriev, V.A. Rubakov, Nucl. Phys. B299 (1988). 67; K. Kajantie, M. Laine, K. Rummukainen, M. Shaposhnikov, Nucl. Phys. B458 (1996) 90, B466 (1996) 189, and references therein.

[5] S. Nosé, J. Chem. Phys. 81, 511 (1984); Mol. Phys. 52 (1984) 255 ; W. G. Hoover, Phys. Rev. A 31 (1985) 1695.

[6] D. Kusnezov, A. Bulgac, W. Bauer, Ann. Phys. 204 (1990) 155; D. Kusnezov, Phys. Lett. 166A 315 (1992).

[7] K. Aoki, D. Kusnezov, Phys. Lett. B477 (2000) 348.

[8] K. Aoki, D. Kusnezov, hep-ph/0002160.

[9] K. Aoki, D. Kusnezov, Phys. Lett. A265 (2000) 250.

[10] H. Kaburaki, M. Machida,Phys. Lett. A181 (1993) 85; S. Lepri, R. Livi, A. Politi, Phys. Rev. Lett. 78 (1997) 1896; Europhys. Lett. 43 (1998) 271.

[11] K. Aoki, D. Kusnezov, Phys. Rev. Lett. 86 (2001) 4029-4033.

[12] J.R. Dorfman, E.G.D. Cohen, Phys. Rev. Lett. 25 (1970) 1257; M.H. Ernst, E.H. Hauge, J.M.J van Leeuwen, Phys. Rev. Lett. 25 (1970) 1254, Phys. Rev. A (1971) 2055; Y. Pomeau, P. Résibois, Phys. Rep. 19 (1975) 63.

[13] C.S. Kim, J.W. Dufty, Phys. Rev. A40 (1989) 6723; N. Nishiguchi, Y. Kawada, T. Sakuma, J. Phys. Cond. Matt. 4 (1992) 10227 ;

[14] G. Aarts, J. Smit, Phys. Lett. 393B (1997) 393; Nucl. Phys.B511 (1998) 451.

[15] K. Kawasaki and J. D. Gunton, Phys. Rev. A 8, 2048 (1973); D.Evans, H.J.M.Hanley,Phys. Lett. 80A (1980) 175.

[16] J.P.Ryckaert, A.Bellemans, G.Ciccotti, G.V.Paolini, Phys. Rev. Lett. 60 (1988) 128; S. Rastogi, N. Wagner, S. Lustig, J. Chem. Phys.104 (1996) 9234; G. Marcelli, B.D.Todd, R. Sadus, Phys. Rev. E63 (2001) 021204. 